\title{
APPLICATION OF OPTIMIZATION ALGORITHMS TO NONLINEAR MPC
}

\author{
Frode Martinsen * Lorenz T. Biegler ${ }^{* *}$ Bjarne A. Foss * \\ * Department of Engineering Cybernetics, NTNU, 7491 Trondheim, \\ Norway. Fax (+47) 73594399 \\ E-mail:frode.martinsen@itk.ntnu.no,bjarne.a.foss@itk.ntnu.no \\ ** Department of Chemical Engineering, Carnegie Mellon University, \\ Pittsburgh, PA 15213. Fax (+1) 4122687139 \\ E-mail: biegler@cmu.edu
}

\begin{abstract}
This paper investigates application of SQP optimization algorithms to nonlinear model predictive control. It considers feasible vs. infeasible path methods, sequential vs. simultaneous methods and reduced vs. full space methods. The suitable choices between these various strategies are assessed informally through a case study.
\end{abstract}

Keywords: model predictive control, optimization

\section{INTRODUCTION}

Nonlinear model predictive control (NMPC) is a control strategy where application of nonlinear optimization methods is essential. This paper is application oriented, and contributes to the practical knowledge of implementation of NMPC. The paper focuses on application of SQP optimization algorithms in NMPC, but emphasizes that appropriate model discretization is essential for the performance. A rule of thumb is that if it is impossible to integrate a model with a certain method, the method cannot be used in NMPC. The paper adresses the differences and similarities between feasible and infeasible path methods, sequential and simultaneous methods and reduced and full space methods. The suitable choices between these various strategies are informally assessed by applying them to a case; a CSTR with first order reaction.

The theory of optimization algorithms is not dependent on how the equality constraints are formed. For instance in optimal control, and in particular in the special case of NMPC, much concern is put into discretization schemes for the nonlinear equality constraints. These equality constraints result from a continuous-time nonlinear dynamical system repeated over a time horizon $P$. Three major variants are usually considered to handle unstable modes; orthogonal collocation, multiple shooting and single shooting (possibly with a variable grid). Ascher et al. (1995) discuss the general benefits of these approaches, and Barclay et al. (1998) discusses this in conjunction with SQP algorithms. Vassiliadis (1993) addresses the dynamic optimization of general DAE systems. These approaches seek to find formulations of the equality constraints that are less hard to satisfy, while simultaneously reducing the discretization error.

Nonlinear inequality constraints may be introduced for stability purposes in NMP C (Scokaert et al. (1999), Chen and Allgöwer (1998b), Chen and Allgöwer (1998a)). These references extends earlier work (Li et al. (1990), de Oliveira and Biegler (1995)), which are concerned with nominal stability. Hence, termination prior to convergence of the optimizer cannot guarantee stability unless the equality constraints are satisfied.

The immediate answer to the need for early termination is single shooting, i.e., solve the model at each iteration with an initial value solver. Single shooting algorithms progress towards a solution by iterating between solving the model and solving a reduced size optimization problem. Due to this, single shooting is said to be a sequential method. Single shooting produces a reduced gradient problem in the free variables to be solved at each NMPC iteration. Maintaining feasibility of nonlinear inequalities involving dependent variables can then be obtained by use of FSQP' (Lawrence and Tits 2000). Single shooting may be costly if evaluation of the problem functions is costly, e.g. if an implicit discretization scheme must be applied. In addition single shooting lacks robustness when applied to unstable systems (Ascher et al. 1995), section 4.1 and 4.6.2.

To solve optimization problems with stabilizing endpoint constraints simultaneous methods must be applied. End-point constraints make the problem a twopoint boundary value problem (TPBVP) which in general cannot be resolved with single shooting. Simultaneous methods do not solve the model at each iteration. Instead a simultaneous search for a model solution and optimal point is carried out. Multipleshooting and orthogonal collocation, possibly on finite elements (Finlayson 1980), are the most widely used simultaneous methods. Since simultaneous methods do not solve the model at each iteration, they cannot guarantee stability in the nominal stability setting of dual-mode or quasi-infinite horizon NMPC if terminated prior to convergence. Note that the results reported in (Bock et al. 2000) show that termination prior to convergence in multiple shooting may be 
viable for some applications. Decomposition strategies for orthogonal collocation on finite elements have been considered by (Cervantes and Biegler 2000) and (Biegler et al. 2001).

For convenience, define the superscript notation $z^{k}=$ $\left\{z_{k}\right\}_{k \subset \mathbb{N}}$ for an entity $z$ indexed by $k$. The nonlinear MPC problem with $\operatorname{dim}\left(u_{k}\right)=n_{u}$ and $\operatorname{dim}\left(x_{k}\right)=n_{x}$

$$
\begin{array}{ll}
\min _{x, u ; k} & \frac{1}{2}\left(\sum_{k=0}^{P-1}\left\|x_{k+1}\right\|_{Q}^{2}+\sum_{k=0}^{M}\left\|u_{k}\right\|_{R}^{2}\right) \\
\text { s.t. } & c_{\mathcal{E}, k}=c_{\mathcal{E}}\left(x^{k}, u^{k}\right)=0 \\
& x^{k} \in \mathbb{X} \times \cdots \times \mathbb{X} \\
& u^{k} \in \mathbb{U} \times \cdots \times \mathbb{U}
\end{array}
$$

with $M<P$ is considered in this paper. The equality constraints in (1) are formed by assuming an appropriate discretization scheme of the continous time model constraint $f(x, u ; t)=0$ repeated over the horizon $P$ along with a suitable parametrization of the control profile. Endpoint constraints or augmentation of the objective may be included to guarantee nominal stability of the MP C algorithm, see (Mayne et al. 2000). Observe that reference tracking and non-zero set-points can be handled in this framework with minor modifications. It is assumed that sufficiently smooth 1 st principles state-space models with measured states, analytic 1st order derivatives are used and that

$$
(\mathcal{X}, \mathcal{U})=(\mathbb{X} \times \cdots \times \mathbb{X}, \mathbb{U} \times \cdots \times \mathbb{X})
$$

can be described by bounds.

The paper continues with a conceptual comparison between single shooting and reduced Hessian methods in section 2. Simulation results follow in section 3 . Discussion and conclusions to the paper follow in sections 4 and 5 .

\section{OPTIMIZATION METHODS}

First SQP methods in general are considered. The MPC problem (1) can be restated as a general nonlinear programming (NLP) problem:

$$
\begin{array}{cc}
\min _{x} & f(x) \\
\text { s.t. } & c_{\mathcal{E}}(x)=0 \\
& c_{\mathcal{I}}(x) \leq 0
\end{array}
$$

where $f:{ }^{n} \rightarrow, c_{\mathcal{E}}:{ }^{n} \rightarrow{ }^{m}$ and $c_{\mathcal{I}}:{ }^{n} \rightarrow^{p}$ where $n=n_{x} P+n_{u} M, m=n_{x}(P+1)$ and $p=2 n$ (assuming upper and lower bounds on $\left(x_{k}, u_{k}\right)$ over the horizons $P$ and $M$ ). The Jacobian matrix of the equality constraints is denoted $A_{k}^{T}=A\left(x_{k}\right)^{T}=$ $\left[\nabla c_{\mathcal{E}}^{1}\left(x_{k}\right), \nabla c_{\mathcal{E}}^{2}\left(x_{k}\right), \cdots, \nabla c_{\mathcal{E}}^{m}\left(x_{k}\right)\right]$ where $c_{\mathcal{E}}^{i}\left(x_{k}\right)$ is the $i$-th component of the vector $c\left(x_{k}\right)$. The matrix $G\left(x_{k}\right)$ made up of $A_{k}^{T}$ and the gradients of the active inequality constraints is assumed to have full column rank. The null-space of $G\left(x_{k}\right)^{T}$ defines the tangent space to the equality and active inequality constraints at $x_{k}$. Denote $H \mathcal{L}_{k}$ the Hessian of the Lagrangian function $\mathcal{L}\left(x_{k}, \lambda_{\mathcal{E}, k}, \lambda_{\mathcal{I}, k}\right)=f\left(x_{k}\right)+\lambda_{\mathcal{E}, k}^{T} c_{\mathcal{E}}\left(x_{k}\right)+$ $\lambda_{\mathcal{I}, k}^{T} c_{\mathcal{I}}\left(x_{k}\right)$ where $\lambda_{\mathcal{E}, k}$ and $\lambda_{\mathcal{I}, k}$ are the multiplier vectors. We assume strong second order sufficient conditions, i.e. that $x^{*}$ is an isolated minimum of the $\operatorname{NLP}(2)$ and that $\lambda_{\mathcal{E}}^{*}$ and $\lambda_{\mathcal{I}}^{*}$ are unique.
In $S Q P$, a sequence of subproblems are solved, where the model is linearized and a quadratic model of the Hessian of the Lagrangian is formed. This gives a quadratic programming problem to be solved at each iteration of the SQP algorithm:

$$
\begin{array}{cl}
\min _{d_{k}} & \nabla f\left(x_{k}\right)^{T} d_{k}+\frac{1}{2} d_{k}^{T} B_{k} d_{k} \\
\text { s.t. } & \nabla c_{\mathcal{E}}\left(x_{k}\right)^{T} d_{k}+c_{\mathcal{E}}\left(x_{k}\right)=0 \\
& \nabla c_{\mathcal{I}}\left(x_{k}\right)^{T} d_{k}+c_{\mathcal{I}}\left(x_{k}\right) \leq 0
\end{array}
$$

where $B_{k} \succ 0$ usually is an approximation to $H \mathcal{L}_{k}$. The solution $d_{k}$ to (3) is a search direction. The SQP algorithm searches along $d_{k}$ for a new iterate $x_{k+1}$ that gives a reduction in a merit function. The merit function $\phi_{\nu}$ is needed to give convergence to a point satisfying the strong 2 nd order assumptions from any starting point under certain additional assumptions.

\subsection{Reduced gradient methods}

The gp (3) can be resolved in the full space of free and independent variables, or in the reduced space of free variables by a suitable elimination of variables. Elimination of variables exploits that if $n_{x} P-$ $n_{u} M>n_{u} M$ and that $n_{u} M$ is small, the reduced subproblem for the null-space step will be small (but dense). In the full space the sparsity of both the Hessian (which commonly requires analytic Hessians) and the Jacobian can be exploited to yield fast solutions (Rao et al. 1998). This section shows that a reduced gradient approach can be derived by following two different strategies. The first uses a sequential approach, see e.g. (de Oliveira and Biegler 1995), while the second follows the simultaneous null-space approach (Nocedal and Wright 1999), (Biegler et al. 1995).

2.1.1. Sequential approach ( $S S Q P) \quad$ By iterating the model over the horizon $P$, the transformation $x^{k}=$ $\Psi\left(x_{0}, u^{k}\right)$ allows the equivalent form which is essentially a projection onto the subspace $\mathcal{U}$. The sequential approach solves the model at each iteration, i.e. the qp-subproblem is solved following a feasible path strategy. The algorithm evolves the model to get $x^{k}=\Psi\left(x_{0}, u^{k}\right)$. Then the algorithm solves the a qp for a direction $d_{k}^{u}$, giving $u^{k+1}$ by a line search, which again is used to evolve the model giving $x^{k+1}$ and so on.

In S SQP the second order derivatives of the model are neglected. This sacrifices the quadratic convergence of Newtons method, but it gives a positive definite Hessian. This is also known as the Gauss-Newton method, which will deteriorate to linear convergence if the projected contributions of the model are significant (Biegler 2000). Since the Hessian $H \mathcal{L}^{*}$ is positive definite, linear convergence results. Otherwise, near the solution quadratic convergence of S SQP may occur, if no state variable bounds are active at the solution.

2.1.2. Reduced Hessian approach (rSQP) In the null-space method a decomposition is applied to the KKT conditions to eliminate variables. It can be shown 
that the SSQP approach coincide with the $r$ SQP approach for the choice $B_{k}=\operatorname{diag}\left(\nabla_{x x}^{2} f_{k}, \nabla_{u u}^{2} f_{k}\right)$ and by decomposing $A_{k}$ into range and null space according to the natural partiotioning given by the states and controls.

In comparing the two approaches observe that the sequential approach maintains feasibility of all iterates, while $r$ SQP searches for feasibility and optimality simultaneously. In addition the sequential method solves the model at each iteration, while $r S Q P$ solves the model only at the solution $x^{*}$. Also note that the sequential approach only handles initial value problems (IVP), i.e. $x_{P} \in \Omega_{x}$ cannot be guaranteed since it implements a shooting strategy in evolving the model over the horizon. The endpoint constraint changes the problem into a boundary value problem (BVP) which must be handled by simultaneous strategies. Therefore the sequential approach is limited to open-loop stable and non-stiff systems. In fact, the stability of the algorithm requires an infinite prediction horizon which is intractable unless the step length can be increased to infinity, see (de Oliveira 1994) and (Chen and Allgöwer 1998a) for details.

There are a number of commercial routines available for both dense and sparse algebra that can be applied. Decomposition strategies for sparse matrices are implemented in the Harwell subroutine libraries MA2 8 and MA 48 (Harwell Laboratory 1995). Alternatives to computing the Jacobian by analytic partial derivatives is by perturbation or by forward differences. This will have a significant impact on the computational demands, see the results in section 3.2.

\section{SIMULATIONS}

NMPC was implemented on a simple case with three different optimization methods. The first is a basic full space $S Q P$ method. The second is the reduced Hessian method $r S Q P$, and the third is the sequential method SSQP. The case is a CSTR with first order reaction. The CSTR example was thoroughly explored by application of various discretization methods and finite difference approximations to the Jacobian.

\subsection{Implementation issues}

The basic SQP full-space method and $r$ SQP were implemented with the common $l_{1}$-penalty function. SSQP implemented an $l_{1}$-penalty function without penalization of equality constraints, since S SQP always remain feasible with respect to equalities. The line search for all methods is backtracking line search.

The relaxed convergence criteria from (Gill et al. 1981), section 8.2.3, were implemented with tolerance $10^{-5}$ for the basic SQP method and SSQP. In $r S Q P$ the algorithm stops whenever a certain KKT measure is decreased below the tolerance $10^{-5}$. The implementation of $r \mathrm{SQP}$ is generally more carefully performed than the basic SQP and S SQP methods. Hence, the relaxed termination criteria used in basic $\mathrm{SQP}$ and $\mathrm{S} S \mathrm{SP}$ partly compensates for a rudimentary implementation. However, as the discussion in section 2 indicates, the S SQP method may show linear convergence in certain circumstances, and relaxed termination criteria can therefore be of crucial importance in production codes as well.

For the CSTR case the model was discretized with explicit and implicit Euler, Lobatto IIIC and ordinary Runge-Kutta 4. The Jacobian matrices with associated almost block-diagonal (ABD) structure for the selected discretization methods. The CSTR case was implemented with both analytic Jacobian and finite difference approximations of the Jacobian. Finite differences were considered for both the full Jacobian (a dense matrix) and the elements along the block diagonal (a sparse matrix). In S SQP analytic and finite difference Jacobians were implemented. I.e. the sensitivity matrix $\mathcal{S}$ was approximated directly by finite difference perturbations of the simulator. The CSTR case was investigated with different sampling rates and prediction and move horizons.

The SSQP method was implemented as a GaussNewton method with analytic 2nd derivatives of the objective, while $r S Q P$ was implemented with BFGS updates.

\subsection{Case: CSTR}

The case is the following isothermal CSTR with 1st order reaction from (Matsuura and Kato 1967) also investigated by (de Oliveira 1994)

$$
\begin{aligned}
& \frac{d x_{1}}{d t}=u_{1}+u_{2}-k_{1} \sqrt{x_{1}} \\
& \frac{d x_{2}}{d t}=\left(C_{B_{1}}-x_{2}\right) \frac{u_{1}}{x_{1}}+\left(C_{B_{2}}-x_{2}\right) \frac{u_{2}}{x_{1}}-\frac{k_{2} x_{2}}{\left(1+x_{2}\right)^{2}}
\end{aligned}
$$

with parameter values $k_{1}=0.2, k_{2}=1, C_{B_{1}}=24.9$ and $C_{B_{2}}=0.1$. For $\left(u_{1}, u_{2}\right)=(1,1)$ the CSTR has three equilibrium points at $x_{1}=100, x_{2} \in$ $(0.633,2.72,7.07)$, with the middle equilibrium point being unstable, and the others stable. The system (4) was discretized with a time step $h$, prediction horizon $P$, move horizon $M$ and simulated for $N_{M P C}$ samples, i.e. the NMPC problem is repeatedly solved $N_{M P C}$ times. At time step 10 the process experiences a $+50 \%$ step in $C_{B_{1}}$ which is seen by the NMPC algorithm through the feedback only. The weights are $Q=10 I_{n_{x}}$ and $R=I_{n_{u}}$ in equation (1) and deviation from stationary values is penalized. Here the control objective is to keep the states and controls at their initial values $x=(100,0.633)$ and $u=(1,1)$.

The physical bounds $\left(x_{i}, u_{i}\right) \geq 0$ are imposed over the horizons. The SQP-algorithms were initialized with the output from the previous call for each NMPC iteration. Note that integral action is not implemented. This is justified by that only a comparison of the optimization methods is investigated, and it is expected that introducing integral action will not influence this comparison. A representative simulation result is shown in Figure 1. The process was simulated by MATLAB's ode 45 in all cases. 

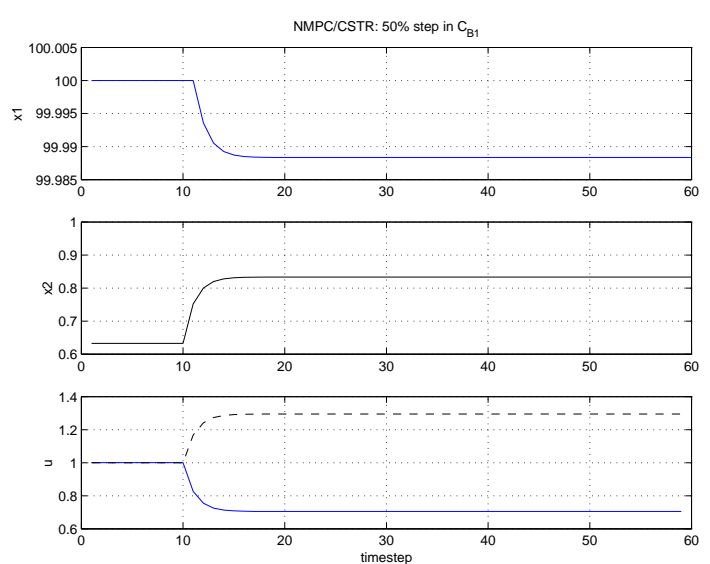

Fig. 1. NMPC of CSTR. The figure shows typical results for NMPC of the CSTR case with $h=$ 1 . The various discretization methods produced nearly identical results. In the lower subfigure the solid curve is for $u_{1}$ and the dashed curve is for $u_{2}$. The step in $C_{B_{1}}$ enters at time step 10 , and this drives the state $x_{2}$ away from the equilibrium. If the control inputs are kept at the equilibrium input $[1,1]$, the system will settle at an equilibrium point at $x=(100,15.94)$.

Note that with the given initial conditions and the given disturbance, this case is pathological with respect to inequality constraints, i.e. the active set is empty and unaltered throughout the horizon $P$.

The computations were implemented in MATLAB with the qp-routines available as mex/dll-files on a Compaq Deskpro EN / Pentium II / 450MHz / 128Mb RAM running Windows NT4.0. Computational results are shown in Tables $1-3 . h / P / M$ are the sampling time, prediction and move horizons. The \# vars. tot/dep/free are the total, dependent and free number of variables. Note that there are no active inequality constraints in this case. The Jacobian is either analytic or approximated by finite differences: In mode fd1 only elements along the block diagonal were approximated, while in mode fd2 the full (dense) Jacobian was approximated. Obj is the objective value measured by summing the deviations of actual prosess outputs and implemented control actions over the NMPC horizon $N_{M P C}$. CPU time is the time measured by MATLAB's cputime command from start to end of the main NMP C loop. The results for S SQP with ode45 with $h=2$ showed some ripple that were caused by the relaxed termination criteria. The results in Tables 1-3 are summarized in the following conclusions:

- $S S Q P$ is sensitive to the choice between implicit and explicit integration methods, while both basic SQP and $r$ SQP is insensitive to this.

- Finite difference approximations of the full Jacobian in basic SQP and $r$ SQP should be avoided.

- Reduced gradient methods are superior for a large number of variables with few degrees of freedom.

Note that a consequence of the first item is that simultaneous SQP methods can have fewer variables when implemented with implicit discretization methods. Here the step length $h$ can be increased beyond the stability limit of explicit methods (but not beyond reasonable accuracy). Since the basic SQP and SSQP methods are approximately similar in implementation complexity, SSQP should be chosen when explicit discretization schemes suffice. In the face of more challenging processes reduced Hessian methods are superior provided that the assumption that there are few degrees of freedom continue to hold. This assumption commonly holds in NMPC.

\section{DISCUSSION}

In this paper different MPC strategies were implemented on a case and the computational load and quality of the results were investigated. From tables 1-3 it is observed that among the different NMPC methods, the rSQP method are preferable in view of computational time if implicit discretization methods are required. In NMPC computational time is limited, and feasibility of intermediate iterates is essential for stability (Mayne et al. 2000), (Scokaert et al. 1999). Generally, SQP involves an adaptive subproblem, i.e. its computational time is not deterministic. Consequently, in NMPC feasible path SQP methods are preferred since they allow termination prior to convergence (Mayne 1997). Such methods must solve the model constraints at each SQP iteration, which may be time consuming if the model is represented with an implicit discretization scheme. Hence, S SQP becomes computationally demanding if implicit discretization methods are applied, whereas simultaneous $S Q P$ methods perform equally well regardless of whether implicit or explicit discretization scheme are applied. Both methods require that the selected discretization scheme is appropriate, i.e. if the model cannot be simulated with a given method, it cannot be expected that the optimization algorithms perform well either.

Feasibility with respect to inequality constraints is easier to achieve. FSQP' (Lawrence and Tits 2000) and rFSQP (Martinsen and Foss 2001) maintain feasibility with respect to inequality constraints, and asymptotic feasibility with respect to nonlinear equality constraints by combining these with an exact penalty function and an arc search. The feasible path SSQP method is expected not to perform well in the presence of strong nonlinearities (Ascher et al. 1995). This is contradictory to the needs for NMP C; e.g. problems with strong nonlinearities and trajectory tracking (Qin and Badgwell 2000).

\section{CONCLUSION}

The practical considerations discussed in this paper explore the choices an engineer must take if he wants to implement NMPC at a given process. The interplay between discretization methods and optimization algorithms has been investigated through a case study.

First the engineer must select an appropriate discretization scheme. If he chooses an explicit dis- 
Table 1. Nonlinear MPC on a CSTR: Basic SQP method.

\begin{tabular}{|c|c|c|c|c|c|}
\hline \multirow{2}{*}{$\begin{array}{l}\text { Discretization } \\
\text { method }\end{array}$} & \multirow{2}{*}{$\begin{array}{l}\text { Horizons } \\
h / P / M\end{array}$} & \multirow{2}{*}{$\begin{array}{c}\text { \# vars. } \\
\text { tot/dep/free }\end{array}$} & \multirow{2}{*}{$\begin{array}{c}\text { Jacobian } \\
\text { analytic/fd } 1 / \mathrm{fd} 2\end{array}$} & \multicolumn{2}{|c|}{ Results } \\
\hline & & & & Obj & CPU time \\
\hline Explicit Euler & $2 / 6 / 5$ & $22 / 12 / 10$ & analytic & 33.55 & $\overline{5.9 \mathrm{~s}}$ \\
\hline Explicit Euler & $1 / 12 / 10$ & $44 / 24 / 20$ & analytic & 28.33 & $19 \mathrm{~s}$ \\
\hline Implicit Euler & $2 / 6 / 5$ & $22 / 12 / 10$ & analytic & 31.41 & $9.5 \mathrm{~s}$ \\
\hline Implicit Euler & $1 / 12 / 10$ & $44 / 24 / 20$ & analytic & 27.85 & $45 \mathrm{~s}$ \\
\hline Lobatto IIIC & $2 / 6 / 5$ & $34 / 24 / 10$ & analytic & 32.37 & $7.3 \mathrm{~s}$ \\
\hline Lobatto IIIC & $2 / 6 / 5$ & $34 / 24 / 10$ & fd1 & 32.35 & $22 \mathrm{~s}$ \\
\hline Lobatto IIIC & $2 / 6 / 5$ & $34 / 24 / 10$ & $\mathrm{fd} 2$ & 32.35 & $25 \mathrm{~s}$ \\
\hline Lobatto IIIC & $1 / 12 / 10$ & $68 / 48 / 20$ & analytic & 28.07 & $35 \mathrm{~s}$ \\
\hline Lobatto IIIC & $1 / 12 / 10$ & $68 / 48 / 20$ & fd1 & 28.06 & $96 \mathrm{~s}$ \\
\hline Lobatto IIIC & $1 / 12 / 10$ & $68 / 48 / 20$ & $\mathrm{fd} 2$ & 28.06 & $222 \mathrm{~s}$ \\
\hline RK4 & $2 / 6 / 5$ & $70 / 60 / 10$ & analytic & 32.40 & $14.1 \mathrm{~s}$ \\
\hline RK4 & $2 / 6 / 5$ & $70 / 60 / 10$ & fd1 & 32.39 & $30 \mathrm{~s}$ \\
\hline RK4 & $2 / 6 / 5$ & $70 / 60 / 10$ & $\mathrm{fd} 2$ & 32.40 & $117 \mathrm{~s}$ \\
\hline RK4 & $1 / 12 / 10$ & $140 / 120 / 20$ & analytic & 28.05 & $119 \mathrm{~s}$ \\
\hline RK4 & $1 / 12 / 10$ & $140 / 120 / 20$ & fd1 & 28.04 & $338 \mathrm{~s}$ \\
\hline RK4 & $1 / 12 / 10$ & $140 / 120 / 20$ & $\mathrm{fd} 2$ & 28.05 & $1136 \mathrm{~s}$ \\
\hline
\end{tabular}

Table 2. Nonlinear MPC on a CSTR: sSQP method.

\begin{tabular}{|c|c|c|c|c|c|}
\hline \multirow{2}{*}{$\begin{array}{l}\text { Discretization } \\
\text { method }\end{array}$} & \multirow{2}{*}{$\begin{array}{l}\text { Horizons } \\
h / P / M\end{array}$} & \multirow{2}{*}{$\begin{array}{c}\text { \# vars. } \\
\text { tot/dep/free }\end{array}$} & \multirow{2}{*}{$\begin{array}{c}\text { Jacobian } \\
\text { analytic/fd }\end{array}$} & \multicolumn{2}{|c|}{ Results } \\
\hline & & & & Obj & CPU time \\
\hline Explicit Euler & $2 / 6 / 5$ & $22 / 12 / 10$ & analytic & 33.18 & $4.4 \mathrm{~s}$ \\
\hline Explicit Euler & $2 / 6 / 5$ & $22 / 12 / 10$ & $\mathrm{fd}$ & 33.54 & $5.7 \mathrm{~s}$ \\
\hline Explicit Euler & $1 / 12 / 10$ & $44 / 24 / 20$ & analytic & 28.15 & $13.1 \mathrm{~s}$ \\
\hline Explicit Euler & $1 / 12 / 10$ & $44 / 24 / 20$ & $\mathrm{fd}$ & 28.33 & $19.4 \mathrm{~s}$ \\
\hline Implicit Euler & $2 / 6 / 5$ & $22 / 12 / 10$ & analytic & 31.41 & $33 \mathrm{~s}$ \\
\hline Implicit Euler & $2 / 6 / 5$ & $22 / 12 / 10$ & $\mathrm{fd}$ & 31.41 & $131 \mathrm{~s}$ \\
\hline Implicit Euler & $1 / 12 / 10$ & $44 / 24 / 20$ & analytic & 27.85 & $94 \mathrm{~s}$ \\
\hline Implicit Euler & $1 / 12 / 10$ & $44 / 24 / 20$ & $\mathrm{fd}$ & 27.83 & $745 \mathrm{~s}$ \\
\hline Lobatto IIIC & $2 / 6 / 5$ & $34 / 24 / 10$ & analytic & 32.37 & $49 \mathrm{~s}$ \\
\hline Lobatto IIIC & $2 / 6 / 5$ & $34 / 24 / 10$ & $\mathrm{fd}$ & 32.36 & $192 \mathrm{~s}$ \\
\hline Lobatto IIIC & $1 / 12 / 10$ & $68 / 48 / 20$ & analytic & 28.07 & $118 \mathrm{~s}$ \\
\hline Lobatto IIIC & $1 / 12 / 10$ & $68 / 48 / 20$ & $\mathrm{fd}$ & 28.07 & 1121s \\
\hline RK4 & $2 / 6 / 5$ & $70 / 60 / 10$ & analytic & 32.40 & $4.3 \mathrm{~s}$ \\
\hline RK4 & $2 / 6 / 5$ & $70 / 60 / 10$ & $\mathrm{fd}$ & 32.40 & $10.0 \mathrm{~s}$ \\
\hline RK4 & $1 / 12 / 10$ & $140 / 120 / 20$ & analytic & 28.05 & $12.2 \mathrm{~s}$ \\
\hline RK4 & $1 / 12 / 10$ & $140 / 120 / 20$ & $\mathrm{fd}$ & 28.05 & $58 \mathrm{~s}$ \\
\hline ode45 & $2 / 6 / 5$ & $22 / 12 / 10$ & $\mathrm{fd}$ & 23.03 & $36 \mathrm{~s}$ \\
\hline ode 45 & $1 / 12 / 10$ & $44 / 24 / 20$ & $\mathrm{fd}$ & 28.49 & $149 \mathrm{~s}$ \\
\hline
\end{tabular}

Table 3. Nonlinear MPC on a CSTR: rSQP method.

\begin{tabular}{|c|c|c|c|c|c|}
\hline \multirow{2}{*}{$\begin{array}{l}\text { Discretization } \\
\text { method }\end{array}$} & \multirow{2}{*}{$\begin{array}{l}\text { Horizons } \\
h / P / M\end{array}$} & \multirow{2}{*}{$\begin{array}{c}\text { \# vars. } \\
\text { tot/dep/free }\end{array}$} & \multirow{2}{*}{$\begin{array}{c}\text { Jacobian } \\
\text { analytic/fd1/fd2 }\end{array}$} & \multicolumn{2}{|c|}{ Results } \\
\hline & & & & Obj & CPU time \\
\hline Explicit Euler & $2 / 6 / 5$ & $22 / 12 / 10$ & analytic & 33.57 & $6.1 \mathrm{~s}$ \\
\hline Explicit Euler & $1 / 12 / 10$ & $44 / 24 / 10$ & analytic & 28.27 & $13.4 \mathrm{~s}$ \\
\hline Implicit Euler & $2 / 6 / 5$ & $22 / 12 / 10$ & analytic & 31.43 & $6.2 \mathrm{~s}$ \\
\hline Implicit Euler & $1 / 12 / 10$ & $44 / 24 / 10$ & analytic & 27.99 & $12.8 \mathrm{~s}$ \\
\hline Lobatto IIIC & $2 / 6 / 5$ & $34 / 24 / 10$ & analytic & 32.37 & $7.1 \mathrm{~s}$ \\
\hline Lobatto IIIC & $2 / 6 / 5$ & $34 / 24 / 10$ & fd1 & 32.35 & $11.5 \mathrm{~s}$ \\
\hline Lobatto IIIC & $2 / 6 / 5$ & $34 / 24 / 10$ & $\mathrm{fd} 2$ & 32.35 & $17.3 \mathrm{~s}$ \\
\hline Lobatto IIIC & $1 / 12 / 10$ & $68 / 48 / 20$ & analytic & 28.07 & $17.2 \mathrm{~s}$ \\
\hline Lobatto IIIC & $1 / 12 / 10$ & $68 / 48 / 20$ & $\mathrm{fd} 1$ & 28.05 & $39 \mathrm{~s}$ \\
\hline Lobatto IIIC & $1 / 12 / 10$ & $68 / 48 / 20$ & $\mathrm{fd} 2$ & 28.06 & $95 \mathrm{~s}$ \\
\hline RK4 & $2 / 6 / 5$ & $70 / 60 / 10$ & analytic & 32.40 & $9.1 \mathrm{~s}$ \\
\hline RK4 & $2 / 6 / 5$ & $70 / 60 / 10$ & $\mathrm{fd} 1$ & 32.39 & $19 \mathrm{~s}$ \\
\hline RK4 & $2 / 6 / 5$ & $70 / 60 / 10$ & $\mathrm{fd} 2$ & 32.40 & $71 \mathrm{~s}$ \\
\hline RK4 & $1 / 12 / 10$ & $140 / 120 / 20$ & analytic & 28.05 & $23 \mathrm{~s}$ \\
\hline RK4 & $1 / 12 / 10$ & $140 / 120 / 20$ & $\mathrm{fd} 1$ & 28.04 & $58 \mathrm{~s}$ \\
\hline RK4 & $1 / 12 / 10$ & $140 / 120 / 20$ & $\mathrm{fd} 2$ & 28.05 & $781 \mathrm{~s}$ \\
\hline
\end{tabular}

cretization scheme, he can choose between sequential or simultaneous optimization methods. Sequential methods are easy to implement, while simultaneous methods are harder to implement. This applies in particular to reduced Hessian methods which may be quite sophisticated. If implicit discretization methods must be applied, the performance of SSQP deteriorates while simultaneous $\mathrm{SQP}$ does not degrade. 


\section{REFERENCES}

Ascher, Uri M., Robert M. M. Mattheij and Robert D. Russell (1995). Numerical solution of boundary value problems for ordinary differential equations. Society for Industrial and Applied Mathematics (SIAM). Philadelphia, PA. Classics in Applied Mathematics.

Barclay, Alex, Philip E. Gill and J. Ben Rosen (1998). SQP methods and their application to numerical optimal control. In: Variational calculus, optimal control and applications (Trassenheide, 1996). pp. 207-222. Birkhäuser. Basel.

Biegler, Lorenz T. (2000). Efficient solution of dynamic optimization and NMPC problems. In: Nonlinear model predictive control (Ascona, 1998). pp. 219-243. Birkhäuser. Basel.

Biegler, Lorenz T., Arturo M. Cervantes and Andreas Wächter (2001). Advances in simultaneous strategies for dynamic process optimization. Technical report. Carnegie Mellon University. Submitted for possible publication.

Biegler, Lorenz T., Jorge Nocedal and Claudia Schmid (1995). A reduced Hessian method for largescale constrained optimization. SIAM J. Optim. 5(2), 314-347.

Bock, Hans Georg, Moritz M. Diehl, Johannes P. Schlöder, Frank Allgöwer, Rolf Findeisen and Zoltan Nagy (2000). Real-time optimization and nonlinear predictive control of processes governed by differential-algebraic equations. In: Prepints: International Symposium on $\mathrm{Ad}$ vanced Control of Chemical Processes (ADCHEM 2000), Pisa, Italy, 2000 (Lorenz T. Biegler, Alessandro Brambilla and Claudio Scali, Eds.). pp. 695-703.

Cervantes, Arturo M. and Lorenz T. Biegler (2000). A stable elemental decomposition for dynamic process optimization. J. Comput. Appl. Math. 120(1-2), 41-57. SQP-based direct discretization methods for practical optimal control problems.

Chen, H. and Frank Allgöwer (1998a). A computationally attractive nonlinear model predictive control scheme with guaranteed stability for stable systems. J. Proc. Cont. 8(5-6), 475-485.

Chen, H. and Frank Allgöwer (1998b). A quasiinfinite horizon nonlinear model predictive control scheme with guaranteed stability. Automatica J. IFAC 34(10), 1205-1217.

de Oliveira, Nuno M. C. and Lorenz T. Biegler (1995). An extension of Newton-type algorithms for nonlinear process control. Automatica J. IFAC 31(2), 281-286.

de Oliveira, Nuno Manuel Clemente (1994). Newtontype algorithms for nonlinear constrained chemical process control. PhD thesis. Carnegie Mellon University. Pittsburgh, PA.

Finlayson, Bruce A. (1980). Nonlinear analysis in chemical engineering. Chemical engineering. McGraw-Hill. New York, NY.

Gill, Philip E., Walter Murray and Margaret H. Wright (1981). Practical optimization. Academic Press Inc. [Harcourt Brace Jovanovich Publishers]. London.
Harwell Laboratory (1995). Harwell Subroutine Library Specifications (Release 12). AEA Technology. Oxfordshire, UK.

Lawrence, Craig T. and André L. Tits (2000). A computationally efficient feasible sequential quadratic programming algorithm. Technical report. Institute for Systems Research, University of Maryland. TR 98-46, Submitted for possible publication in SIAM J. Optim.

Li, Wei Chong, Lorenz T. Biegler, C. G. Economou and M. Morari (1990). A constrained pseudoNewton control strategy for nonlinear systems. Computers Chem. Engng. 14(4/5), 451-468.

Martinsen, Frode and Bjarne A. Foss (2001). A feasible reduced Hessian SQP method. Technical report. Department of Engineering Cybernetics, NTNU, Norway. ITK-2001-5-W. Submitted for possible publication in SIAM Journal on Optimization.

Matsuura, T. and M. Kato (1967). Concentration stability of the isothermal reactor. Chem. Eng. Sci. 22, 171-184.

Mayne, David Q. (1997). Nonlinear model predictive control : An assessment. In: $C P C$-V : Proceedings of the Fifth International Conference on Chemical Process Control, Tahoe City, CA., 1996 (J. C. Kantor, C. E. Garcia and B. Carnahan, Eds.). AIChE symposium series ; no. 316. CACHE. pp. 217-231.

Mayne, David Q., James B. Rawlings, Christopher V. Rao and Pierre Scokaert (2000). Constrained model predictive control: Stability and optimality. Automatica J. IFAC 36(6), 789-814.

Nocedal, Jorge and Stephen J. Wright (1999). Numerical optimization. Springer-Verlag. New York.

Qin, S. Joe and Thomas A. Badgwell (2000). An overview of nonlinear model predictive control applications. In: Nonlinear model predictive control (Ascona, 1998) (Frank Allgöwer and Alex Zheng, Eds.). pp. 128-145. Birkhäuser.

Rao, C. V., S. J. Wright and J. B. Rawlings (1998). Application of interior-point methods to model predictive control. J. Optim. Theory Appl. 99(3), 723-757.

Scokaert, Pierre O. M., David Q. Mayne and James B. Rawlings (1999). Suboptimal model predictive control (feasibility implies stability). IEEE Trans. Auto. Cont 44(3), 648-654.

Vassiliadis, V. (1993). Computational solution of dynamic optimization problems with general differential-algebraic constraints. $\mathrm{PhD}$ thesis. University of London. U.K. 